\title{
Properties which cannot be Explained by Planetary Nebulae's Progenitors
}

\author{
Noam Soker
}

University of Haifa at Oranim

Stellar binary companions account for bipolar PNe $\left(\sim 11 \%\right.$ of all $\left.\mathrm{PNe}^{1}\right)$, and some ellipticalls $\left(22 \%^{2}\right)$. The rest of axisymmetrical PNe $(40 \%$ to $60 \%$ of all $\mathrm{PNe})$ are due to substellar objects (planets and brown dwarfs) ${ }^{3}$. This classification of axisymmetrical PNe suggests that substellar objects are commonly present within several $\times \mathrm{AU}$ around main sequence stars, and that several substellar objects must be present around most main sequence stars ${ }^{3}$. Some substellar and low mass stellar companions will enter the primary envelope only as the primary reaches the upper AGB. Thus, the early mass loss episode will be spherical, leading to the formation of a spherical halo around an elliptical inner region. Gas giant planets and brown dwarfs close to the primary, will not allow Earth-like planets to have stable orbits. Systems with no Jupiter-like planets will allow Earth-like planets to be present. These stars will form spherical PNe (10\%-20\% of all PNe, including spherically ejected PNe that have been deformed by the ISM through which they move ${ }^{4}$ ). Systems with substellar objects at large separation, as Jupiter in the solar system, will also allow Earth-like planets to be present. These systems will form PNe with spherical halo. Therefore, life may have been present in planets around the central stars of round PNe and elliptical PNe with round halos.

I hope that during the next IAU Symposium on PNe time will be allocated to discuss the interaction of PNe with the ISM, the influence of substellar objects on PNe, and the effects of wide stellar companions.

\section{REFERENCES}

Schwarz, H. E., \& Corradi, R. L. M. 1995, Asymmetrical Planetary Nebulae, eds. A. Harpaz and N. Soker, p. 113.

Yungelson, L. R., Tutukov, A. V., \& Livio, M., 1993, ApJ, 418, 794.

Soker, N. 1996, ApJL, 460, L53.

Soker, N. 1997, preprint. 